\title{
Nationwide Epidemiology of Melioidosis in Sri Lanka
}

\section{E M Corea}

Melioidosis has been compared with an iceberg, since the majority of cases are hidden. National surveillance was instituted with a network of microbiology laboratories and a standard case definition, laboratory work up procedure and questionnaire. Primary isolation relied on conventional culture. Suspected isolates were referred to the reference laboratory for bacteriological identification and confirmation by real time PCR assays for the LpxO gene. Demographic and clinical data of culture positive patients were analysed.

A total of 250 cases were recorded between 2006 and May 2017. Number increased annually with 103 (41\%) detected in 2016. Males predominated, 179 (71.6\%) being male. Sixteen were children. The age range was wide (2-92y) reflecting ubiquity of exposure to soil in the population. Commonest age group was middle-aged (41-60y), corresponding to likelihood of soil exposure and age of onset of diabetes. Majority $(201 / 250,80 \%)$ lived in rural areas. All provinces were affected with the highest number from the Western $(n=88)$, North Western $(n=54)$ and Eastern $(n=33)$ Provinces. There were no cases in the cooler hill country where the main crop is not rice, but tea/rubber. Eighty seven patients presented between May/July and 81 between November/January, the two monsoonal periods (67\%) with an increase in case load in June/July 2016 after heavy rains and flooding in May.

Only 44 patients were farmers. There was representation of every population group. While diabetes was the predominant risk factor $(n=163,65.2 \%)$, organ disease, alcohol excess and thalassaemia were also predisposing factors. Melioidosis was seen in healthy adults and children (33/250, 13.2\%). Clinical presentations included community acquired sepsis and pneumonia, superficial and deep abscesses and septic arthritis. Central nervous system and genitourinary infection was seen. One had endocarditis. Mortality was $20.4 \%(51 / 250)$.

The majority of isolates belong to the YLF clade but 38 strains belonged to the BTFC (Australian) type. A total of 108 isolates were genotyped and 46 sequence types (STs) were identified, 40 being novel at the time of submission. ST1137 was commonest ST in Sri Lanka $(n=18)$. Sri Lanka has the largest representation of all the South Asian countries in the international B.pseudomallei database, accounting for more than $2.4 \%$ of the entire database. The large number of STs (46) suggests that microevolution is occurring through genetic rearrangement.

Melioidosis is endemic in Sri Lanka with a wide geographic and demographic distribution. Improved diagnosis has led to reduced mortality. There is an urgent need to extend surveillance of melioidosis to under-resourced parts of the country and to populations at high risk.

Data presented in this abstract has been published in greater detail in Corea EM, Silva ADD, Thevanesam V. Melioidosis in Sri Lanka. Tropical Medicine and Infectious Disease. 2018; 3(1):22.

Department of Microbiology, Faculty of Medicine, University of Colombo, Colombo, Sri Lanka

Address for correspondence: Dr Enoka Corea, Department of Microbiology, Faculty of Medicine, University of

Colombo, Sri Lanka.+94 777808439 Email: enokacorea@hotmail.com iD https://orcid.org/0000-0003-1450-2098 